\title{
Micromixing enhancement by turbulence: Application to multifunctional heat exchangers
}

\author{
S. Ferrouillat ${ }^{\text {a }}$, P. Tochon ${ }^{a}$, H. Peerhossaini ${ }^{b, *}$ \\ ${ }^{a}$ Commissariat of Atomic Energy-GRETh, 17 Avenue des Martyrs, F-38054 Grenoble Cedex 9, France \\ ${ }^{\mathrm{b}}$ Laboratoire de Thermocinétique, CNRS-UMR 6607 Ecole Polytechnique de l'Université de Nantes, \\ Rue Christian Pauc, BP 50609, F-44306 Nantes Cedex 3, France
}

\begin{abstract}
Compact heat exchangers are well-known for their ability to transfer large amounts of heat while retaining low volume and weight. This paper studies the use of this device as a chemical reactor, generally called a heat exchanger reactor (HEX reactor). Indeed, the question arises: can these geometries combine heat transfer and mixing in the same device? Such a technology would offer many advantages, such as better reaction control (through the thermal aspect), improved selectivity (through intensified mixing, more isothermal operation and shorter residence time, and sharper residence-time distribution), byproduct reduction, and enhanced safety.

Several geometries of compact heat exchanger based on turbulence generation are available. This paper focuses on two types: offset strip fins (OSFs) and metallic foams. Our main objective is to contribute to the estimation of micromixing generated by these geometries by using an experimental method based on a unique parallel-competing reaction scheme proposed by Villermaux et al. The micromixing time, estimated according to the incorporation model, lets us compare the micromixing levels generated by duct channel, OSFs and metallic foams at volume flow rates ranging from 1 to $3501 \mathrm{~h}^{-1}$. The metallic foam concept is found to be very efficient in micromixing enhancement. Furthermore, OSFs make it possible to generate micromixing levels ranging between the duct channel and metallic foam level. Moreover, the results show that the fin micromixing level increases with fin thickness and ligament diameter. Finally, in an HEX reactor application, the residence time of chemical reactants must be considered in order to choose the best geometry for intensifying mass and heat transfer.
\end{abstract}

Keywords: Compact heat exchanger reactor; Micromixing; Metallic foam; Offset strip fins; Continuous system; Multifunctional heat exchanger

\section{Introduction}

Initially developed in the 1940s in the aerospace industry to provide compact, light, highly efficient heat exchangers for gas/gas applications, the compact plate-fin heat exchanger has potential applications as a reactor in the chemical industry: heat exchanger reactors (HEX reactors) or multifunctional heat exchangers (MHE). This technology offers many advantages: better reaction control (from the thermal point of view), improved selectivity (through intensified mixing, more isothermal operation and shorter residence time, and sharper residencetime distribution), reduction of byproducts, and greater safety. Indeed, although many traditional designs such as stirred tanks

\footnotetext{
* Corresponding author. Tel.: +33 2406831 39; fax: +332 40683141 E-mail address: hassan.peerhossaini@univ-nantes.fr (H. Peerhossaini).
}

already incorporate heat transfer, in these devices there is a significant distance between the heat-transfer device and the site of the chemical reaction. The aim of the HEX reactor is to eliminate this distance by supplying or removing heat almost as rapidly as it is absorbed or generated by the reaction. The heat-transfer performance comparison of different MHE reactors in Table 1 shows the major advantages of MHE [1]. Indeed, thanks to its advanced heat-transfer performance and unlike the other technologies, this process should be able to realize strong exothermal reactions without thermal runaway.

However, mixing and in particular micromixing have an important influence on product quality in industrial reactions such as polymerization or precipitation. More precisely, industrial reactions depend on mixing at the molecular scale if their characteristic reaction times are close to the micromixing time. Thus, in order to improve such MHE reactors, it is important to characterize mixing at the molecular scale. 
Table 1

Heat exchange capability for different reactors

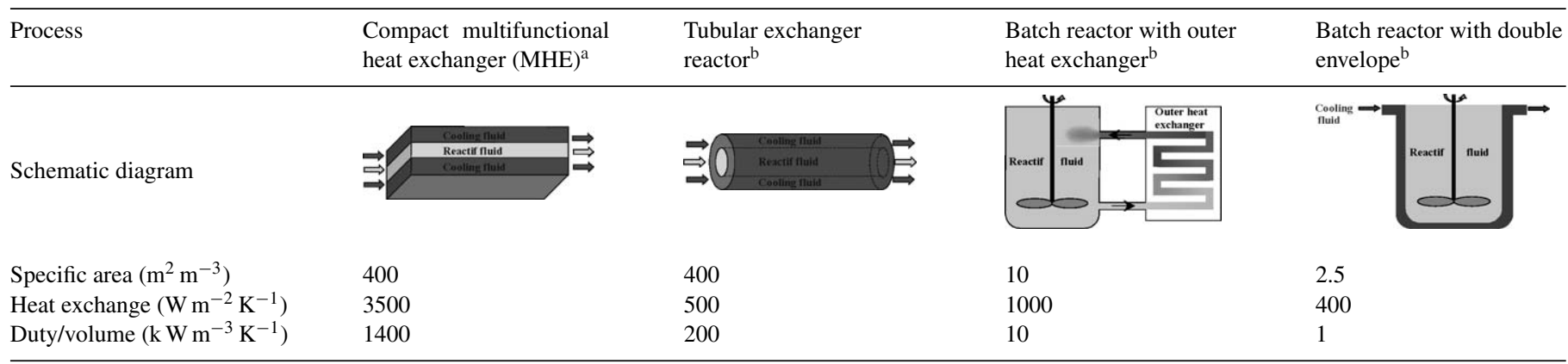

${ }^{\text {a }}$ Results based on metallic foam, 45 ppi at $R e=1000$.

b Trambouze and Euzen [1].

Several chemical methods have been proposed to measure the micromixing generated by different geometries inserted in a continuous process [2-4]. The available literature reports many thermohydraulic data on compact heat exchangers. However, micromixing has almost never been studied in the same process. Therefore, the goal of this work is to quantify the micromixing level generated inside an MHE.

This paper presents an experimental micromixing study realized on different geometries a priori in order to intensify mass transfer in the process. It starts by validating the chemical method used. Then an estimate of the micromixing time generated by different geometries using the incorporation model is presented. In addition to a simple empty duct channel, two other geometries were studied:

- offset strip fins (OSF)

- metallic foams

This paper evaluates micromixing in each geometry and compares their performance. It also provides a database of the micromixing performances of several geometries so that the design engineer can choose the most appropriate geometry to intensify mass transfer and thus optimise a MHE.

\section{A physicochemical experimental method for determination of micromixing}

Experimental data obtained with test reactions show that physical parameters such as impeller speed or feed location can influence the distribution of reaction products in batch processes. Fournier et al. [5] reviewed the literature on ways to measure micromixing. Two main chemical methods have been detailed:

- consecutive competing reactions (diazo coupling test) developed by Bourne et al. [6,7]:

$$
\begin{aligned}
& \mathrm{A}+\mathrm{B} \rightarrow \mathrm{R} \\
& \mathrm{R}+\mathrm{B} \rightarrow \mathrm{S}
\end{aligned}
$$

- parallel-competing reactions (iodide-iodate test) developed by Villermaux et al. [5,8]:

$$
\begin{aligned}
& \mathrm{A}+\mathrm{B} \rightarrow \mathrm{R} \\
& \mathrm{C}+\mathrm{B} \rightarrow \mathrm{S} .
\end{aligned}
$$

According to Guichardon [8,9], the iodide-iodate reaction is preferable for convenience in experimentation and analysis. Consequently, in this work, the micromixing level estimate generated by several geometries was tested using Villermaux et al.'s parallel-competing reaction scheme.

Villermaux et al. proposed a unique parallel-competing reaction system with $\mathrm{A}, \mathrm{B}, \mathrm{R}$ and $\mathrm{S}$ as $\mathrm{H}_{2} \mathrm{BO}_{3}{ }^{-}, \mathrm{H}^{+}, \mathrm{H}_{3} \mathrm{BO}_{3}$ and $\mathrm{I}_{2}$, respectively; $\mathrm{C}$ denotes the mixture of $5 \mathrm{I}^{-}+\mathrm{IO}_{3}{ }^{-}$:

$\mathrm{H}_{2} \mathrm{BO}_{3}^{-}+\mathrm{H}^{+} \rightarrow \mathrm{H}_{3} \mathrm{BO}_{3}$ quasi - instantaneous,

$5 \mathrm{I}^{-}+\mathrm{IO}_{3}^{-}+6 \mathrm{H}^{+} \rightarrow 3 \mathrm{I}_{2}+3 \mathrm{H}_{2} \mathrm{O}$ very fast

The redox Eq. (2) is fast (in the same range as the micromixing process), but is much slower than the neutralisation reaction (1).

\subsection{Segregation index}

The test procedure consists in adding in a stoichiometric defect, a small quantity of sulphuric acid, to a mixture of iodate, iodide and borate ions. The amount of iodine produced depends on the efficiency of the micromixing process when acid is a limiting reactant, so that the selectivity of iodine is a measure of the segregation state of the fluid. The fraction of acid used in the formation reaction of iodine is a convenient measure of the product distribution and makes possible the calculation of the selectivity of acid $Y=2 n_{\mathrm{I}_{2}} / n_{\mathrm{H}_{0}}$ and the index of molecularscale segregation:

$X_{\mathrm{S}}=\frac{Y}{Y_{\mathrm{ST}}}$,

where $Y_{\mathrm{ST}}=3 \mathrm{C}_{\mathrm{IO}_{3,0}-} /\left(6 \mathrm{C}_{\mathrm{IO}_{3,0} 0^{-}}+\mathrm{C}_{\mathrm{H}_{2} \mathrm{BO}_{3,0}{ }^{-}}\right)$is the selectivity at total segregation. It should be mentioned that this normalized segregation index $X_{\mathrm{s}}$, whose value lies between 0 and 1 , 
decreases with increasing micromixing level. The advantage of $X_{\mathrm{S}}$ is that it is closely related to the ratio of two characteristic times: reaction time and micromixing time.

The iodine $I_{2}$ formed in reaction (2) can react further with iodide ions $\mathrm{I}^{-}$to yield $\mathrm{I}_{3}{ }^{-}$ions according to the quasiinstantaneous equilibrium

$\mathrm{I}_{2}+\mathrm{I}^{-} \rightarrow \mathrm{I}_{3}^{-}$

The $\mathrm{I}_{3}{ }^{-}$concentration can be easily measured by spectrophotometry at $353 \mathrm{~nm}$. Mass balances on iodine atoms yield the expressions

$\mathrm{C}_{\mathrm{I}^{-}}=\left(\mathrm{C}_{\mathrm{I}^{-}}\right)_{0}-\frac{5}{3}\left(\mathrm{C}_{\mathrm{I}_{2}}+\mathrm{C}_{\mathrm{I}_{3}-}\right)-\mathrm{C}_{\mathrm{I}_{3}-}$

$\mathrm{K}_{\mathrm{B}}=\frac{\mathrm{C}_{\mathrm{I}_{3}-}}{\mathrm{C}_{\mathrm{I}_{2}} \mathrm{C}_{\mathrm{I}^{-}}}$

where $K_{\mathrm{B}}$ is the equilibrium constant of reaction (3). By combining these two equations, we obtain a second-order algebraic equation for iodine concentration:

$-\frac{5}{3}\left(\mathrm{C}_{\mathrm{I}_{2}}\right)^{2}+\left[\left(\mathrm{C}_{\mathrm{I}^{-}}\right)_{0}-\frac{8}{3} \mathrm{C}_{\mathrm{I}_{3}-}\right] \mathrm{C}_{\mathrm{I}_{2}}-\frac{\mathrm{C}_{\mathrm{I}_{3}-}}{\mathrm{K}_{\mathrm{B}}}=0$

Resolution of this second-order algebraic equation makes it possible to estimate the iodine concentration and thus the segregation index $X_{\mathrm{S}}$.

\subsection{Micromixing model}

The segregation index is a measure of the influence of hydrodynamics on chemical selectivity. It depends on two processes:

- chemical processes with characteristic times that are directly linked to the kinetics of chemical reactions (1) and (2),

- the physical process of micromixing, with the micromixing time.

For constant initial reactant concentrations, the variation in $X_{\mathrm{S}}$ accurately takes into account the variation in micromixing level, namely the micromixing time. Under these conditions, $X_{\mathrm{S}}$ can be directly compared for different micromixing conditions, allowing comparison of mixer performance. For nonconstant reaction time and micromixing time conditions, interpretation of the results is more complex and a specific micromixing model based on mixing and reaction-coupled processes must be used.

Fournier et al. [5] used the incorporation model to estimate the micromixing time $t_{\mathrm{m}}$. Fig. 1 illustrates this model. Two fluids, 1 and 2 , are considered. The model is based on the assumption that the fresh fluid 2 (sulphuric acid in the present experiments) is divided into aggregates that are progressively invaded by fluid 1 from the external environment, containing iodide and iodate in some basic medium. The characteristic incorporation time is assumed equal to the micromixing time $t_{\mathrm{m}}$. The volume of the aggregate grows as $V_{2}=V_{2.0} g(t)$, where $g(t)$ depends on the incorporation mechanism and is less than or equal to $V_{1,0}+V_{2,0}$. Two empirical evolution models are proposed:

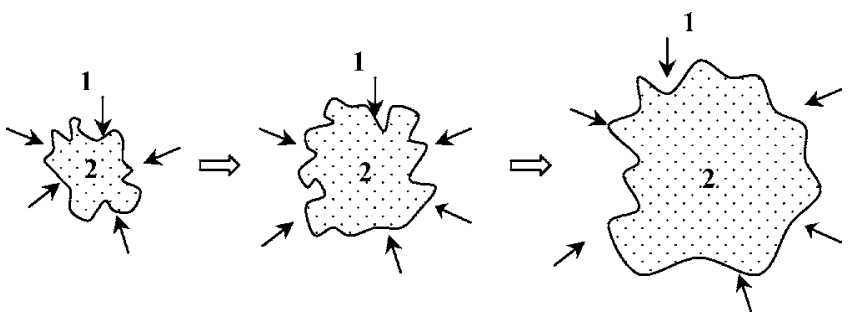

Fig. 1. Schematic diagram of the incorporation model.

- a linear model: $g(t)=1+t / t_{\mathrm{m}}$,

- an exponential model: $g(t)=\exp \left(t / t_{\mathrm{m}}\right)$.

A constant incorporation flow rate leads to a linear relation time, and flow proportional to instantaneous aggregate volume leads to an exponential expression. The reactions are assumed to occur in aggregate 2 , where perfect mixing is assumed achieved. In the reaction volume 2, the concentrations of the $j$ species are given by:

$\frac{\mathrm{dC}_{j}}{\mathrm{~d} t}=\left(\mathrm{C}_{j 1,0}-\mathrm{C}_{j}\right) \frac{1 \mathrm{~d} g}{g} \frac{\mathrm{d} t}{\mathrm{~d} t}+R_{j}$

where subscript 1,0 indicates the surrounding fluid and $R_{j}$ is the specific rate of formation of reactant $j$. From the initial reactant concentrations and an assumed initial segregation index $X_{\mathrm{S}}$, resolving the differential equation system (7) lets us estimate the micromixing time $t_{\mathrm{m}}$.

\section{Experimental test facility}

Experiments were carried out in a test section made of a perspex rectangular duct channel of height $6.35 \mathrm{~mm}$ and width $40 \mathrm{~mm}$ in which various geometries are inserted. Fig. 2 is a schematic diagram of the experimental setup.

The main flow, composed of iodide, iodate and orthoboric acid, is pumped into the test section and sulphuric acid is added by a symmetrical " $\mathrm{T}$ " injection with a syringe pump in the core of the geometry. The symmetrical injection is realized by two pipes of diameter $1 \mathrm{~mm}$. The main flow rate is measured by a rotometer and the main initial volume is temperature-regulated at $25^{\circ} \mathrm{C}$. The concentration of $\mathrm{I}_{3}{ }^{-}$is measured continuously by spectrophotometry at $353 \mathrm{~nm}$ with instantaneous computer data acquisition.

Six geometries (three OSFs and three metallic foams), inserted into the same duct channel of hydraulic diameter $D_{\mathrm{h}}=11 \mathrm{~mm}$, height $6.35 \mathrm{~mm}$ and width $40 \mathrm{~mm}$, were tested. The three OSFs inserted in the duct channel have the characteristics given in Fig. 3 and Table 2; Table 3 and Fig. 4 give the characteristics of the three metallic foams inserted in the duct channel. Different geometries were tested with the reactant concentrations shown in Table 4 (based on Fang and Lee [10]). 


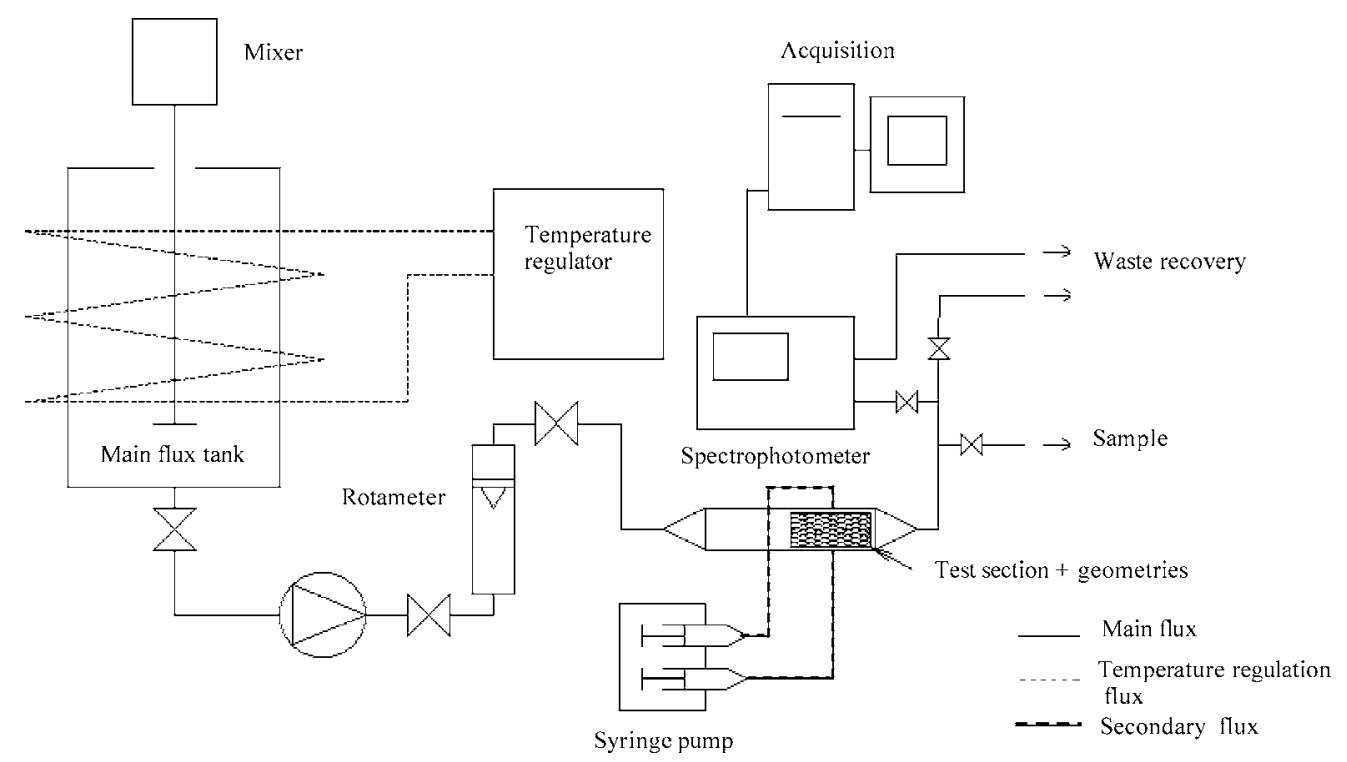

Fig. 2. The experimental setup.
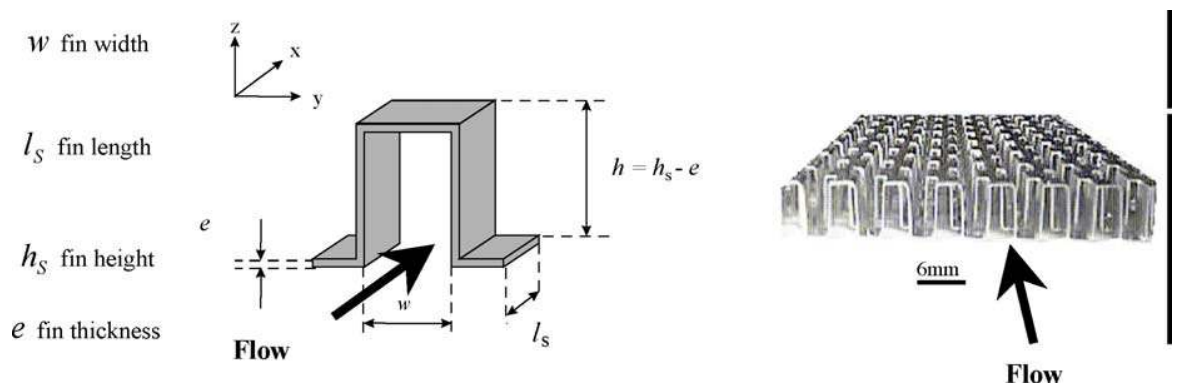

Fig. 3. Offset strip fin geometry.

Table 2

Offset strip fins characteristics

\begin{tabular}{lccc}
\hline Fin type & OSF 1 & OSF 2 & OSF 3 \\
\hline$e(\mathrm{~mm})$ & 0.1 & 0.3 & 0.3 \\
$w(\mathrm{~mm})$ & 1.4 & 3.0 & 1.2 \\
$l_{\mathrm{S}}(\mathrm{mm})$ & 3.2 & 1.6 & 3.2 \\
$h_{\mathrm{S}}(\mathrm{mm})$ & 6.35 & 6.35 & 6.35 \\
Fin number per volume unit $\left(\mathrm{m}^{-3}\right)$ & $35.2 \times 10^{8}$ & $32.8 \times 10^{8}$ & $41.0 \times 10^{8}$ \\
$D_{\mathrm{H}}(\mathrm{mm})$ & 2.2 & 3.5 & 1.9 \\
\hline
\end{tabular}

Where $D_{\mathrm{H}}=4 w h l_{\mathrm{s}} / 2\left(w l_{\mathrm{s}}+h l_{\mathrm{s}}+e h\right)+e w$.

\section{Validation of the physicochemical model}

\subsection{Influence of sulphuric acid injection}

In order to quantify the effect of micromixing alone, it is necessary to work in a medium in which macromixing conditions
Table 4

Reactant concentrations

\begin{tabular}{ll}
\hline Reactant & Concentration $\left(\mathrm{moll}^{-1}\right)$ \\
\hline $\mathrm{H}_{3} \mathrm{BO}_{3}$ & 0.0121 \\
$\mathrm{NaOH}$ & 0.00605 \\
$\mathrm{KIO}_{3}$ & 0.00233 \\
$\mathrm{KI}$ & 0.0116 \\
$\mathrm{H}_{2} \mathrm{SO}_{4}$ & 0.25 or 0.50 \\
\hline
\end{tabular}

are fulfilled. For rapid sulphuric acid injections, the reactant is not well-dispersed in the test section, and macroscopic concentration gradients can influence the results by generating high segregation indices. For this reason, all experiments were carried out with acid injection time slow enough to prevent macro and mesomixing effects. Under these conditions, macromixing effects are negligible and the results of chemical test reactions are

Table 3

Metallic foam characteristics

\begin{tabular}{lllllr}
\hline Foam type & Materials & Grade (constructor) $(\mathrm{ppi})$ & Pore diameter $(\mathrm{mm})$ & Ligament diameter $(\mathrm{mm})$ & ${\text { Specific surface }\left(\mathrm{m}^{2} \mathrm{~m}^{-3}\right)}_{\text {Porosity }(\%)}$ \\
\hline ERG, 20 ppi & Aluminium & 20 & 1.5 & 0.4 & 1720 \\
SCPS, 20 ppi & Copper & 20 & 1.2 & 0.3 & 900 \\
SCPS, 45 ppi & Copper & 45 & 0.8 & 0.2 & 2400 \\
\hline
\end{tabular}




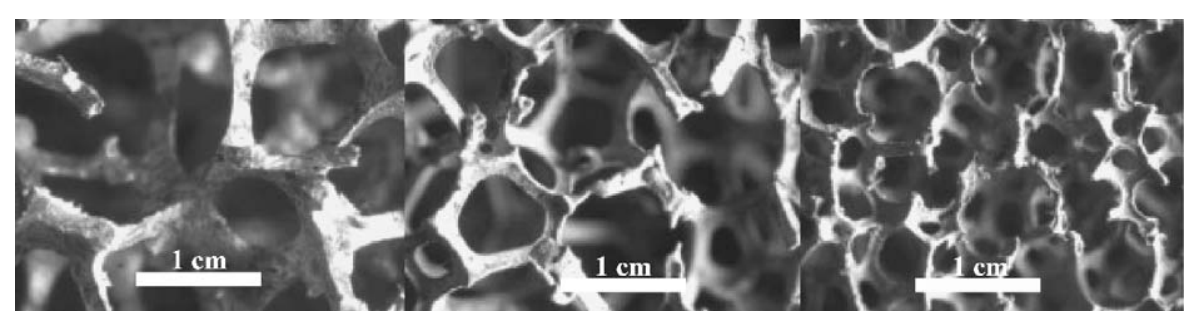

Fig. 4. Metallic foam photography at the same scales (left to right: 20 ppi ERG, 20 ppi SCPS and 45 ppi SCPS).

relevant only to micromixing. For the present continuous analysis, the influence of the ratio between the main flow rate and the secondary flow rate on the index segregation $X_{\mathrm{S}}$ was tested for the worst micromixing operating conditions, namely in the empty duct channel at Reynolds number 550. Fig. 5 shows the decay of the segregation index with the increase of the ratio between the main flow rate and the secondary flow rate, in other words the decrease of the injection flow rate. The ratio for which the segregation index reaches a constant value is called the critical ratio $\left(Q_{\mathrm{A}} / Q_{\mathrm{B}}\right)_{\text {critical }}$. For the present conditions, the critical ratio is near 2500. In all subsequent experiments, the ratio $\left(Q_{\mathrm{A}} / Q_{\mathrm{B}}\right)$ was fixed at 3000 to insure that macromixing plays no significant role during injection.

\subsection{Influence of reactant concentration}

Two different concentrations of sulphuric acid were tested in order to assess their influence on micromixing times. Micromixing analysis depends on two time constants, the characteristic reaction time of the reactions involved and the characteristic micromixing time. Since the chemical systems differ drastically, it is impossible directly to compare the segregation index obtained with these two methods. For this reason, the incorporation model is used to obtain a micromixing time extracted from the segregation index, which depends only on hydrodynamic conditions. Since the experiments were carried out under the same hydrodynamic conditions for both reactant concentrations, the micromixing times obtained should be the same. Fig. 6 shows that for a given flow rate, the micromixing times

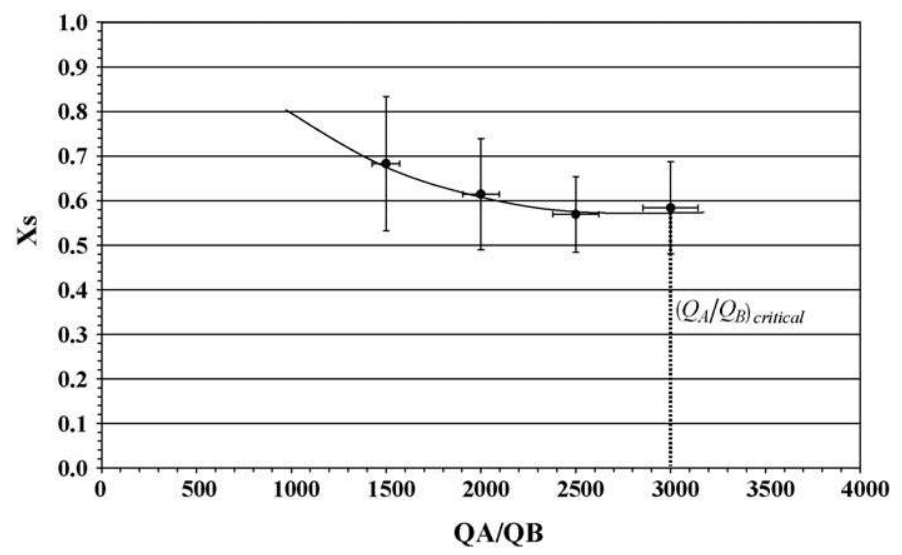

Fig. 5. Segregation index vs. ratio between main flow rate and secondary flow rate (empty duct channel, $R e=550$ ). obtained are of the same order of magnitude, thus verifying that the incorporation model correctly takes into account the effect of the sulphuric acid concentration. Consequently, this method seems well-adapted to comparing micromixing performances generated by different geometries in a continuous process.

\section{Results}

The main objective of this work is to evaluate the micromixing generated by different geometries in order to intensify mass transfer in a MHE. Here we summarize and compare micromixing results obtained for the different geometries studied.

\subsection{Offset strip fins}

Fig. 7 compares micromixing time and flow rate for the empty duct channel and OSFs studied. This figure shows that, whatever the geometry, the micromixing time decreases with the flow rate. It also shows that, for a given flow rate, the empty duct channel is clearly the worst in micromixing performance. OSF 1 generates the same order of micromixing level as the empty duct channel, far below that of the other two fins. This observation can be explained by considering OSF hydrodynamics as similar to that of thick flat plates, as suggested by a previous study [11]. A characteristic size frequently used in this kind of geometry is the fin half thickness. Many authors [12-14] define several flow regimes around thick flat plates. In order to determine the flow regime, a Reynolds number based on the fin half thickness $R e_{\mathrm{e} / 2}$ is defined. These authors agree in proposing three flow regimes,

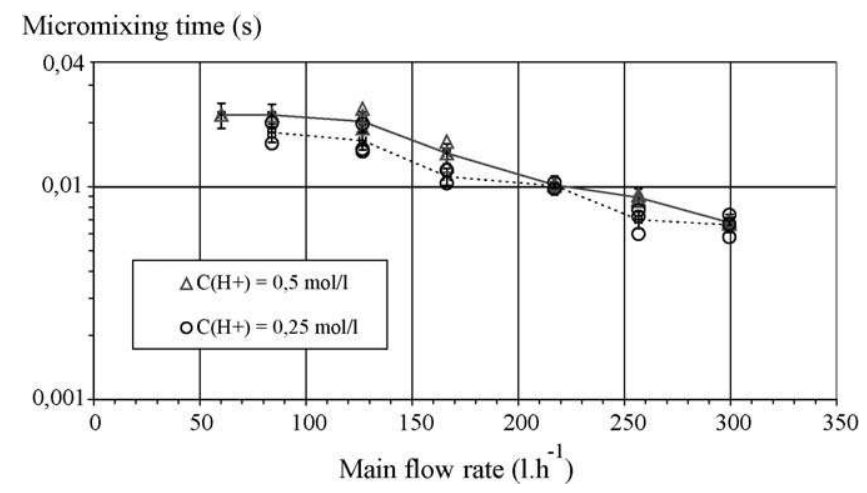

Fig. 6. Comparison of micromixing time with two concentration reactants vs. flow rate. 
Micromixing time (s)

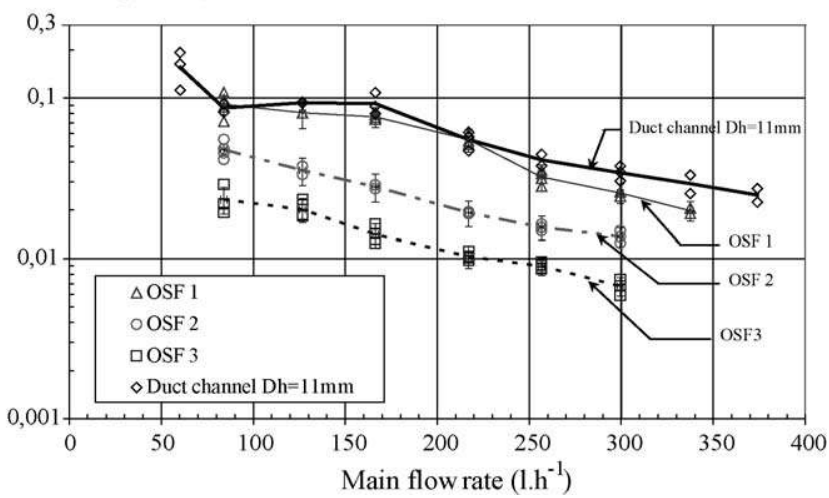

Fig. 7. Micromixing time vs. flow rate for three different offset strip fins and a duct channel.

but the precise Reynolds numbers for the transition regime differ according to author.

The three flow regimes are:

- For $R e_{\mathrm{e} / 2}<200$, boundary-layer separation and reattachment are both laminar but for $R e_{\mathrm{e} / 2}>60$ Bénard-Karman eddies appear in the wake of OSF.

- For $200<R e_{\mathrm{e} / 2}<400$, boundary-layer separation is still laminar but the reattachment becomes turbulent.

- For $R e_{\mathrm{e} / 2}>400$, boundary-layer separation and reattachment are both turbulent.

For this reason (see Fig. 8), for a given velocity, the thicker the fin, the higher the Reynolds number $R e_{\mathrm{e} / 2}$ and consequently the greater the turbulence and micromixing. The range of Reynolds number $R e_{\mathrm{e} / 2}$ is between 5 and 25 for OSF 1,15 and 65 for OSF 2 , and 20 and 75 for OSF 3 . This observation suggests a priori that boundary-layer separation and reattachment are laminar and that only OSF 2 and 3 can generate Bénard-Karman vortex shedding. Consequently, OSF 1 generates no more micromixing than the duct channel because of its small fin thickness. However, Michel [11] showed that upstream conditions strongly influence the transition Reynolds number and flow behaviour, e.g. high-intensity turbulence upstream of the fins produces an

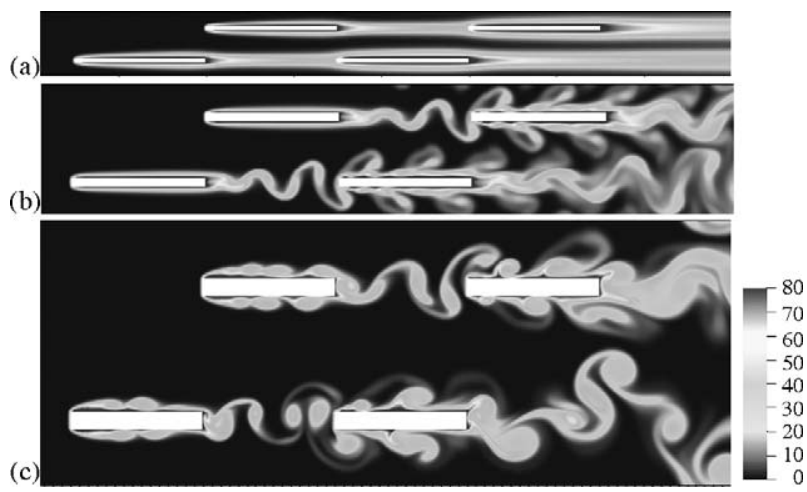

Fig. 8. Temperature visualization of Bénard-Karman vortices generated by different fin thicknesses. Flow Reynolds number is, respectively, $R e=120$ (a); 240 (b); and $360(\mathrm{c})[11]$.

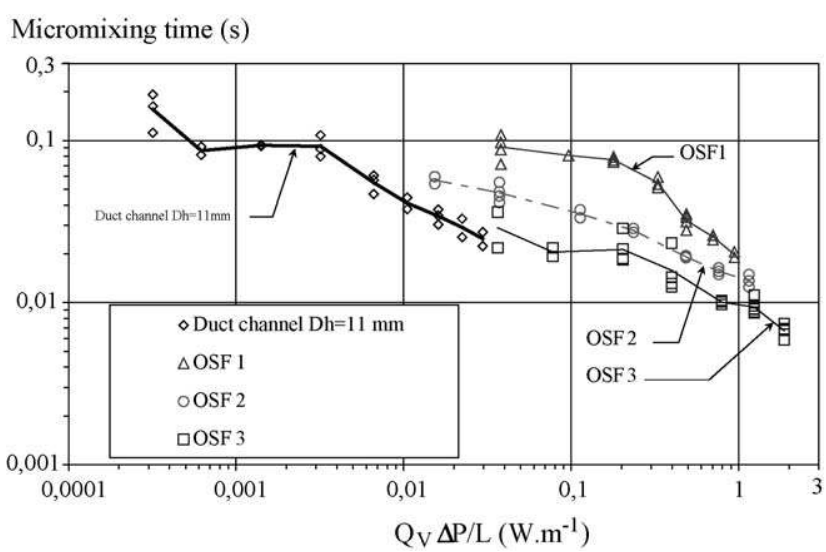

Fig. 9. Micromixing time vs. pumping power for three different offset strip fins and a duct channel.

easier transition to turbulence. Thus upstream conditions can explain the differences between the present results and those in the general fluid mechanics literature.

In addition, OSF 3 generates a better micromixing level than OSF 2 even though they have the same fin thickness. This observation suggests that the increase in number of fins per volume unit, as shown in Table 2, induces large flow shearing and then an increase in turbulence and micromixing. It is thus advisable to use OSFs with the largest fin thickness and greatest number of fins per unit volume in order to generate maximum micromixing.

For MHE applications, one must also consider the pumping power, e.g. the pressure drop, which can be obtained by using the correlation in Manglik and Bergles [15] for OSFs geometries and the correlation in Shah and Bhatti [16] for the empty duct channel. Fig. 9, a plot of micromixing time against pumping power, shows that for the same flow rate, the empty duct channel generates the smallest pressure drop. OSFs seem no more attractive in pumping power than the empty duct channel. This approach also shows that the pumping power needed for different OSFs increases with fin thickness and number of fins per unit volume. It is also interesting to see that the micromixing time seems to converge toward the same values for high pumping-power values. The characteristic geometry has a stronger influence in the laminar regime than in the turbulent regime. Indeed, a suitable characteristic geometry decreases the transition to turbulence. Then, when turbulence is established, the geometry has less impact on micromixing.

\subsection{Metallic foams}

As in the OSFs study, Fig. 10 shows that for metallic foams, micromixing time decreases when the flow rate increases, suggesting that the micromixing level depends on flow velocity or turbulence intensity.

First (and unexpectedly), for the same flow rate, micromixing time decreases when the metallic foam pore diameter increases. At first glance this seems contradictory, since strong shearing due to a decrease in pore diameter should normally generate high turbulence intensity. However, if one considers metallic foam as an arrangement of vertical and horizontal tubes, as suggested by 


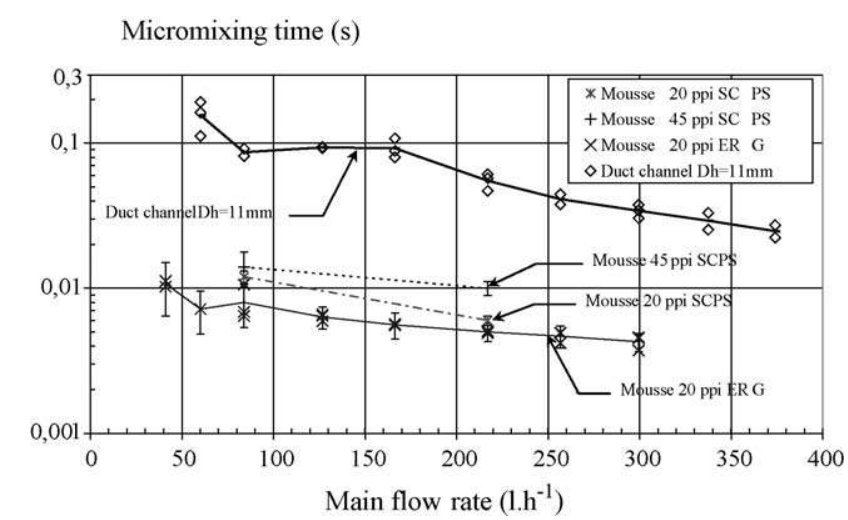

Fig. 10. Micromixing time vs. flow rate for three different metallic foams and a duct channel.

$\mathrm{Lu}$ et al. [17], it is possible to consider a metallic foam flow as an external flow around a tube. For that kind of flow, the suitable characteristic length is then the cylinder diameter or metallic foam ligament diameter. As for OSFs, several flow regimes can be defined using a Reynolds number based on cylinder diameter or metallic foam ligament diameter. With this approach, for the same velocity, the larger the ligament diameter, the higher the turbulence intensity. Thus, for a given flow rate, Fig. 10 shows that micromixing time decreases with increasing metallic foam ligament diameter. These results are therefore in agreement with the hypothesis of external flow around a tube. For the same flow rate, metallic foam seems the best geometry for micromixing. As for OSF, micromixing seems to increase with increasing leadingedge effect. Hydrodynamic flows with OSF or metallic foam are similar and can be considered as external flows around a flat plate or a foam ligament.

With the same approach as in the OSF case, Fig. 11 plots micromixing time against pumping power. Unlike the micromixing level, which increases with ligament diameter, the pressure drop increases with decreasing pore diameter. Indeed, the strong shear level induced by the large number of leading edges generates a high singular pressure drop. Consequently, for the $45 \mathrm{ppi}$ $\mathrm{SCPS}$, the pressure drop is a primary drawback for micromix-

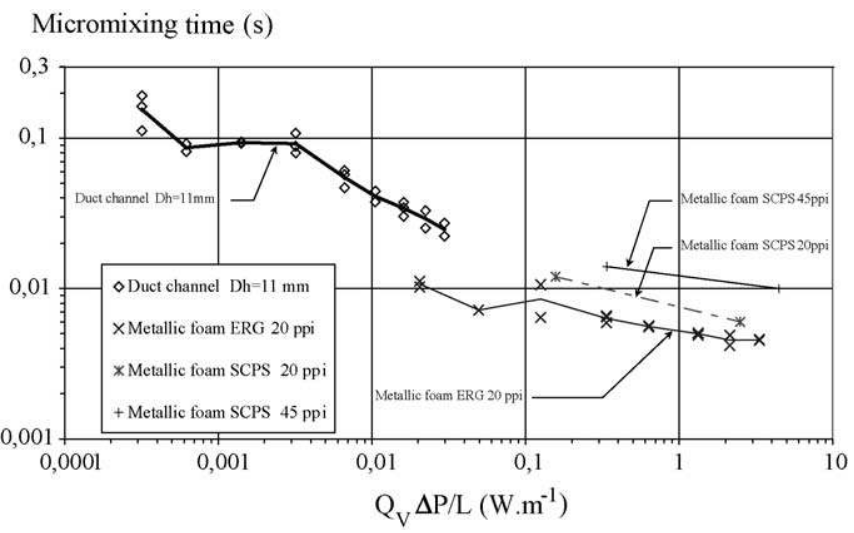

Fig. 11. Micromixing time vs. pumping power for three different metallic foams and a duct channel. ing performance. On the other hand, the 20 ppi ERG, which has the largest pore diameter, generates a smaller pressure drop while producing a high micromixing level; thanks to its larger ligament diameter. The micromixing level reflects the ability of the geometry to convert pressure drop into turbulence rather than into viscous friction. Thus in order to produce high micromixing, a metallic foam with a large ligament diameter is recommended-while taking care of the generated pressure drop.

\section{Concluding remarks}

For optimal use, heat exchanger reactors must enhance both heat and mass transfer. The integration of inserts that can promote turbulence is of prime importance. This study has shown advantages and drawbacks in using OSFs or metallic foams as fin surfaces for heat exchanger reactors. Since thermohydraulic data on compact heat exchangers are available in open literature but micromixing data are very rare, the challenge of this work has been to quantify micromixing level generated inside an MHE. The present work quantitatively measures the micromixing level generated by several geometries on the basis of the unique parallel-competing reaction scheme proposed by Villermaux et al. Micromixing time was estimated based on incorporation model proposed by Fournier et al. [5].

This study showed that for a given flow rate, an empty duct channel generates a reasonable micromixing level without too great pumping power. Nevertheless, the residence time of chemical reactants in an MHE reactor is an important parameter and must be taken into account. When the residence time for $1 \mathrm{~m}$ of an MHE reactor is taken into account, Fig. 12 shows that metallic foam is the geometry that generates the best micromixing level for a given residence time. Its isotropic geometry is also an important advantage in creating homogeneous flow in the MHE reactors (perfect plug flow). Indeed, the reaction medium must be as uniform as possible in order to reduce byproduct formation. Furthermore, metallic foam has a large specific area and could be very interesting as a catalyst medium. The pressure drop seems to be the main drawback of the metallic foams. On the other

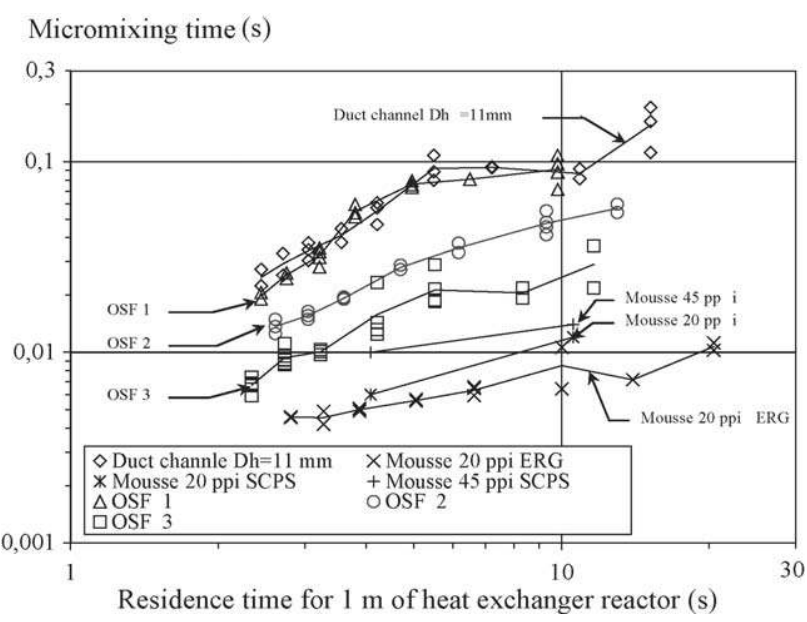

Fig. 12. Micromixing time vs. resident time for $1 \mathrm{~m}$ of a heat exchanger reactor for all geometries studied. 
hand, for a given flow rate, OSFs need less pumping power but generate less micromixing than metallic foams. Moreover, for a given micromixing time, the empty duct channel must work at too high velocity, implying an increase in the volume of the MHE. For a given micromixing level, insert geometries let us reduce the volume of the reactor but lead to a pumping-power increase. Thus, it is necessary to take into account in process design the pumping power required for the system, the process compactness, and the micromixing level required.

This work provides a rich database of micromixing performances of several geometries such as OSFs and metallic foams inserted in a duct channel. Moreover, an experimental micromixing method tested and validated with the incorporation model is now available for testing other geometries, such as vortex generators or microchannels, in order to quantify the micromixing level generated in a continuous process.

\section{Acknowledgements}

This work was partially supported by the "ENERGIE" programme of CNRS and the French Ministry of Research. S.F. acknowledges the financial support of ADEME (French Agency for Energy and the Environment) and CEA (Commissariat of Atomic Energy).

\section{Appendix A. Nomenclature}

\section{$C_{j} \quad$ chemical concentration of species $j, \mathrm{moll}^{-1}$}

$C_{j 1,0}$ initial reactant concentration for surrounding fluid, $\mathrm{moll}^{-1}$

$D_{\mathrm{h}} \quad$ hydraulic diameter, $\mathrm{m}$

$D_{\mathrm{H}} \quad$ offset strip fins hydraulic diameter, $\mathrm{m}$

$e \quad$ offset strip fins thickness, m

$g(t)$ incorporation function

$h_{\mathrm{s}} \quad$ offset strip fins height, $\mathrm{m}$

$K_{\mathrm{B}} \quad$ equilibrium constant, $1 \mathrm{~mol}^{-1}$

$l_{\mathrm{s}} \quad$ offset strip fins length, $\mathrm{m}$

$L \quad$ test section length, $\mathrm{m}$

$Q_{\mathrm{V}} \quad$ volume flow rate, $1 \mathrm{~h}^{-1}$

$\left(Q_{\mathrm{A}} / Q_{\mathrm{B}}\right)$ ratio of main flow rate and secondary flow rate

$\left(Q_{\mathrm{A}} / Q_{\mathrm{B}}\right)_{\text {critical }}$ critical ratio of main flow rate and secondary flow rate

$R_{j} \quad$ specific rate of formation of reactant $j, \operatorname{mol~m}^{-3} \mathrm{~s}^{-1}$

$t_{\mathrm{m}} \quad$ characteristic micromixing time, $\mathrm{s}$

$V_{i} \quad$ aggregate volume of species $i, \mathrm{~m}^{3}$

$V_{i, 0} \quad$ initial aggregate volume of species $i, \mathrm{~m}^{3}$

$w \quad$ width between two offset strip fins, $m$

\section{Greek symbols}

$\triangle P \quad$ pressure drop, $\mathrm{Pa}$

$\varepsilon \quad$ local rate of turbulence energy dissipation, $\mathrm{W} \mathrm{kg}^{-1}$

\section{Subscripts}

$0 \quad$ at initial time

1 surrounding fluid

2 fresh fluid

j reactant

\section{Dimensionless expressions}

Re Reynolds number

$X_{\mathrm{S}}=Y / Y_{\mathrm{ST}}$ segregation index

$Y \quad$ yield

$Y_{\mathrm{ST}} \quad$ yield at total segregation

\section{References}

[1] P. Trambouze, J.P. Euzen, Les réacteurs chimiques de la conception à la mise en œuvre, Éditions Technip, Paris, 2002.

[2] M.C. Fournier 1, L. Falk, J Villermaux, A new parallel-competing reaction system for assessing micromixing efficiency determination of micromixing time by a simple mixing model, Chem. Eng. Sci. 51 (1996) 5187-5192.

[3] J.R. Bourne, H. Maire, Micromixing and fast chemical reactions in static mixers, Chem. Eng. Process 30 (1991) 23-30.

[4] P. Guichardon, L. Falk, Characterisation of micromixing efficiency by the iodide-iodate reaction system. Part I: experimental procedure, Chem. Eng. Sci. 55 (2000) 4233-4243.

[5] M.C. Fournier, L. Falk, J. Villermaux, A new parallel-competing reaction system for assessing micromixing efficiency: experimental approach, Chem. Eng. Sci. 51 (22) (1996) 5053-5064.

[6] J. Baldyga, J.R. Bourne, Simplification of micromixing calculations. I: derivation and application of a new model, Chem. Eng. J. 42 (1989) 83-92.

[7] J.R. Bourne, J. Baldyga, Turbulent Mixing and Chemical Reaction, Wiley, New York, 1999.

[8] P. Guichardon, L. Falk, Characterization of micromixing efficiency by the iodide-iodate reaction system. Part 1: experimental procedure, Chem. Eng. Sci. 55 (2000) 4233-4243.

[9] P. Guichardon, L. Falk, M. Andrieu, Experimental comparison of the iodide-iodate and the diazo coupling micromixing test reactions in stirred reactors, Trans. IchemE. 79 (Part A) (2001) 906-914.

[10] J.Z. Fang, D.J. Lee, Micromixing efficiency in static mixer, Chem. Eng. Sci. 56 (2001) 3797-3802.

[11] F. Michel, Optimisation des échangeurs compacts à ailettes: Étude numérique et expérimentale, Ph.D. thesis, University of Joseph Fourrier, Grenoble, 2003.

[12] T. Ota, Y. Asano, J.I. Okawa, Reattachment length and transition of separated flow over blunt flat plates, Bull. JSME 24 (192) (1981).

[13] J.C. Lane, R.I. Loehrke, Leading edge separation from a blunt plate at low Reynolds number, ASME, J. Fluids Eng. 102 (1980) 494496.

[14] K. Sasaki, M. Kiya, Three-dimensional vortex structure in a leading-edge separation bubble at moderate Reynolds numbers, ASME, J. Fluids Eng. 113 (1991) 405-410.

[15] R.M. Manglik, A.E. Bergles, Heat transfer and pressure drop correlations for rectangular offset strip fin compact heat exchanger, Exp. Therm. Fluid Sc. 10 (1995) 171-181.

[16] R.K. Shah, M.S. Bhatti, Handbook of Single Phase Convective Heat Transfer, John Wiley and Sons, New York, 1987 (Chapters 3, 4).

[17] T.J. Lu, H.A. Stone, M.F. Ashby, Heat transfer in open-cell metal foams, Acta Materialia 46 (10) (1998) 3619-3635. 